Open Access

\title{
Influence of the holding time of the deep cryogenic treatment on the strain hardening behavior of HY-TUF steel
}

\author{
Ahmad Zare ${ }^{*}$, H. Mansouri and S. R. Hosseini
}

\begin{abstract}
Background: The effect of the holding time of the deep cryogenic treatment on the strain hardening of HY-TUF, a low-alloy medium-carbon steel, and a comparison with strain hardening of conventional heat-treated steel were investigated.

Methods: For this purpose, a deep cryogenic treatment was done for different holding times of 12,24 , 48, and $72 \mathrm{~h}$. The metallographic results indicate completion of martensitic transformation; $\eta$-carbide precipitation and decrement of carbon concentration in martensitic matrix happened while carrying out a deep cryogenic process.

Results: Observations of transmission electron microscopy indicate higher density of dislocations within martensite near the cementite compared to that near the $n$-carbides. The tensile flow stress data for this steel was examined in terms of Hollomon equation. The results show that strain hardening of conventional heat-treated steel takes place in two stages.

Conclusions: This evidence is related to the co-deformation of austenite and martensite. The strain hardening takes place in one stage for the deep cryogenic-treated steels, and their strain hardening exponents increase (from $\sim 0.29$ to 0.47) with an increasing holding time up to $48 \mathrm{~h}$. A further increase in the holding time of the deep cryogenic treatment is found not to vary strain hardening exponent. The increase of strain hardening exponent and then observation of plateau in this parameter show an optimum value for the holding time of the deep cryogenic treatment (48 h).
\end{abstract}

Keywords: Strain hardening, Deep cryogenic treatment, Martensite, Austenite, $\eta$-carbide

\section{Background}

Material hardening is generally registered by the true stress-strain relationship gotten through the conventional tension test. The four most commonly used empirical strain hardening laws to fit the tensile data in the area of uniform plastic deformation to empirical equations were suggested by Hollomon (1945), Voce (1948), and Swift (1952). Among these equations, the segment of true stress-strain curve from yielding to ultimate stress for multi-phase steels can truly be matched by the following experimental Hollomon equation:

$$
\sigma=K \varepsilon^{n}
$$

* Correspondence: ahmad273273@gmail.com

Department of Materials Engineering, Maleke-ashtar University of Technology, Isfahan 83145-115, Islamic Republic of Iran where $n$ is the strain hardening exponent, $\varepsilon$ the true strain, $K$ the strength coefficient, and $\sigma$ the true stress. The constant parameters of strain hardening in Eq. 1 consisting of $K$ and $n$ can be measured by drawing the true stress-strain data in a diagram on double natural logarithmic scale and fitting a straight line at it. The intercept at the unit value of the true strain obtains the $K$ magnitude, while the slope of the line gives the $n$ value. It is well known that the strain hardening parameters, $K$ and $n$, are of significant industrial attention. The magnitude of $n$ maintains quantification of the capability to decelerate localization of the plastic deformation of the materials. Materials with a high magnitude of strain hardening exponent are selected for operations, which involve plastic deformation because the higher the magnitude of $n$, the greater is the rate at which the material strain hardens (Meyers and Chawla 1991; Akbarpour

\section{勿 Springer}


and Ekrami 2008). The value of $K$ provides the strength level of material or the magnitude of required forces in plastic deformation (Akbarpour and Ekrami 2008; Hertzberg 1996). Moreover, the unique material strength properties, ultimate tensile strength (UTS) and yield strength (YS), are individually important to consider and control as they influence the behavior of structures during accident in airplanes (Marin 1962). Taken together as the UTS/YS ratio (known as the "strain hardening value" in European practice) and strength difference (UTS-YS), they indicate the ductility capacity of the structural components where they were used (Fridman 1975). It is mentioned that the UTS/YS and UTS-YS can be considered as criteria for strain hardening.

HY-TUF steel is a specific class of low-alloy mediumcarbon steel that derives its strength from martensitic transformation and precipitation of carbides in a quenched and tempered condition. In HY-TUF steel, compared to other low-alloy medium-carbon steels (such as AISI 4340 steel), $\mathrm{C}$ content is maintained at a low level $(<0.26 \mathrm{wt} \%)$; the contents of $\mathrm{Si}$ and $\mathrm{Mn}$ are relatively high ( $3 \mathrm{wt} \%)$, as well as $\mathrm{Ni}(\sim 1.80 \mathrm{wt} \%)$. This design of the composition is considered for HY-TUF steel to get superior toughness and resistance to crack propagation as well as ultra-high strength. In addition, vacuum arc remelting (VAR) is used in the steel-making process of HY-TUF steel to have a clean material with low content of inclusions. HY-TUF steel has aerospace and aircraft applications where lightweight structure with ultra-high strength and toughness are essential and cost is not a major concern such as main landing gear, ultra-sensitive screws, and flexible drive shaft of helicopter (Klopp 1992).

Deep cryogenic treatment is a beneficial way in improving the mechanical properties of steels. Deep cryogenic treatment increases hardness, strength, and toughness and improves abrasive and fatigue wear resistance. Deep cryogenic treatment has been investigated to enhance mechanical properties of steels because it improves the microstructure. This process decreases or eliminates retained austenite (Leskovšek et al. 2006). In addition, a deep cryogenic process with post-tempering treatment causes precipitation of fine $\eta$-carbides from supersaturated martensite. These fine carbides have spherical morphology and a high homogenized distribution (Senthilkumar et al. 2011). The martensite lath is originated during deep cryogenic treatment, distributed more uniformly, and has smaller colonies, because the martensitic transformation is suppressed with the formation of fine $\eta$-carbides, which are coherent with the matrix (Li et al. 2010a, b).

There are generally two atomic mechanisms that describe the formation of $\eta$-carbide during a deep cryogenic process. The first mechanism is based on moving of carbon atoms towards the dislocations during long holding at cryogenic temperatures. The carbon clusters adjacent to the dislocations which are considered as nuclei for the $\eta$ carbide (Li et al. 2010a, b, 2011). Tyshchenko et al. (2010) considered the second hypothesis to interpret the carbon redistribution in the martensitic matrix. The suggested mechanism which was studied by using Mossbauer spectroscopy and internal friction measurements attributed the formation of $\eta$-carbide to capture locked carbon atoms by mobile dislocations. At present, little is understood about the stability of cryo-treated $\eta$-carbide which is a transient type. Recently, Hadi (2014) presented data of differential scanning calorimetry about aging of $\eta$-carbide in the cryo-treated HY-TUF steel. It is mentioned that $\eta$-type carbide is stable during aging at a temperature range of $200-400{ }^{\circ} \mathrm{C}$ and does not transform into more stable carbides such as cementite. This evidence is attributed to the coherency of interphase boundaries of $\eta$-carbide and martensite that was observed by a high-resolution transmission electron microscope.

The literature studies showed that even though the micromechanism behind the strain hardening and its relationship with deep cryogenic-treated (DCT) microstructure have not been clarified, different microstructural observations have been studied in the literatures ( $\mathrm{Li}$ et al. 2010a, b; Akhbarizadeh et al. 2009; Amini et al. 2012; Chi et al. 2010; Jaswin and Lal 2011; Li et al. 2010a, b; Koneshlou et al. 2011). So the present research is undertaken to investigate the influence of the holding time of the deep cryogenic treatment on the strain hardening behavior of HY-TUF steel. The micromechanisms of strain hardening were realized by explaining the strain hardening behavior in terms of Hollomon equation. The strain hardening of conventional heat-treated (CHT) and DCT steels are also compared. In all cycles, the austenitizing and tempering parameters were considered constant to evaluate the effect of the holding time of the deep cryogenic treatment on the strain hardening phenomenon.

\section{Methods}

The material used in this study was a VAR HY-TUF steel in the form of 100-mm-diameter forged bars. The chemical composition of this low-alloy medium-carbon steel conforms to that of AMS 6425 (Unterweiser 1982) and is given in Table 1 .

Schematic illustration of the cycles of the cryogenic heat treatment is shown in Fig. 1. Five groups of samples were prepared by means of the following schedules of cryogenic heat treatment: (a) austenitizing at $850{ }^{\circ} \mathrm{C}$ for $1 \mathrm{~h}$; (b) quenching into warm oil at $100{ }^{\circ} \mathrm{C}$; (c)

Table 1 Chemical composition of the steel (wt\%)

\begin{tabular}{lllllllllllll}
\hline $\mathrm{C}$ & $\mathrm{Mn}$ & $\mathrm{Si}$ & $\mathrm{Ni}$ & $\mathrm{Co}$ & $\mathrm{Cr}$ & $\mathrm{Mo}$ & $\mathrm{V}$ & $\mathrm{Cu}$ & $\mathrm{Al}$ & $\mathrm{Ti}$ & $\mathrm{Fe}$ & $\mathrm{Ms}$ \\
\hline 0.25 & 1.40 & 1.60 & 1.80 & - & 0.30 & 0.45 & - & - & - & - & Balance & 357 \\
\hline
\end{tabular}




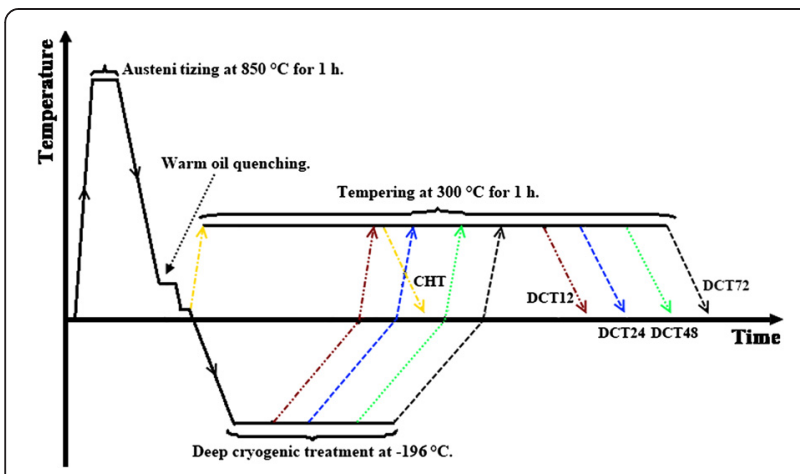

Fig. 1 Schematic diagram of cryogenic heat cycle used in this study

subsequent deep cryogenic treatment at $-196{ }^{\circ} \mathrm{C}$ for different times of $12,24,48$, and $72 \mathrm{~h}$; and (d) subsequent tempering at $300{ }^{\circ} \mathrm{C}$ for $1 \mathrm{~h}$. As shown in Fig. 1, DCT samples were labeled as DCT12, DCT24, DCT48, and DCT72 (the numbers are representative of the holding time of the deep cryogenic process). It should be mentioned that CHT steel did not passed through the deep cryogenic treatment. The cryogenic treatments of the samples were performed by placing them in a liquid nitrogen chamber. Temperature of the samples was monitored by a K-type thermocouple.

For observing retained austenite, the specimens were ground, polished, and etched with a solution which has the chemical compositions of " $1 \mathrm{~g}$ picric acid, $5 \mathrm{ml} \mathrm{HCl}$, and $100 \mathrm{ml}$ ethanol" (Girault et al. 1998). Figure 2 shows an example of the microstructures where retained austenite is clearly distinguished from the other phases. Metallographic samples of the studied steels were also etched (after the removal of all traces of the earlier etchant) with " $100 \mathrm{ml} \mathrm{H} \mathrm{H}_{2} \mathrm{O}, 10 \mathrm{~g} \mathrm{~K}_{3} \mathrm{Fe}(\mathrm{CN})_{6}$, and $10 \mathrm{~g}$ $\mathrm{NaOH}$ " reagent to observe carbides which are shown in Fig. 3. After etching with this reagent, the carbides appear bright, whereas the remaining phases (martensite and austenite) are dim (Girault et al. 1998). At least 20 areas on the surfaces of the polished and etched samples were examined at different magnifications to acquire statistically reliable data. The average of the carbide volume fraction was reported for each sample. The existence of retained austenite was also revealed by means of X-ray diffraction (XRD) with a Philips PW3040 unit and using a copper target. The working voltage and the working current were $40 \mathrm{kV}$ and $30 \mathrm{~mA}$, respectively. Specimens for transmission electron microscopy (TEM) were first cut to a thickness of $300 \mu \mathrm{m}$ and subsequently ground to a thickness of $100 \mu \mathrm{m}$. Then, 3-mm disks were punched out and were polished in a FISHCHION 110 twin jet polisher at an operating voltage of $75 \mathrm{~V}$. The samples were observed in a PHILIPS CM300 transmission electron microscope.

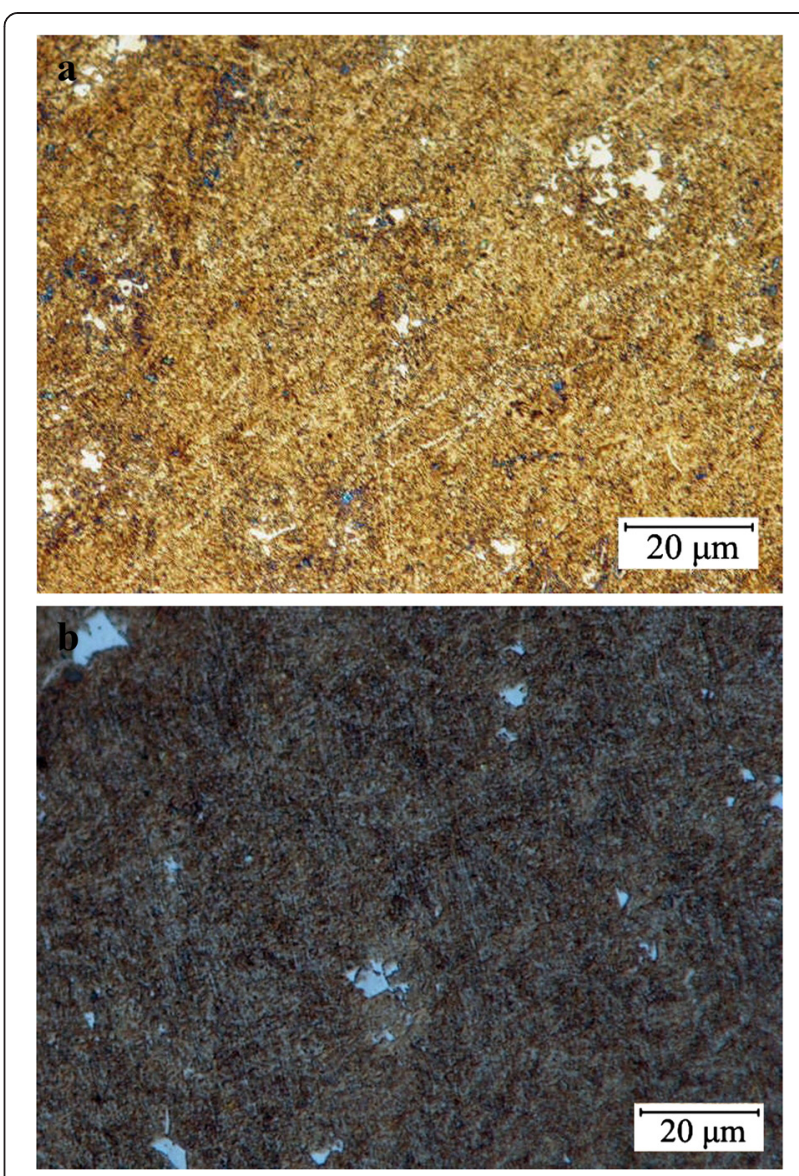

Fig. 2 Optical microstructures of $\mathbf{a}$ CHT and $\mathbf{b}$ DCT48 steels showing retained austenite (light regions)

Tensile samples were cut from the forged bars of HYTUF steel by a wire cut machine. The strain hardening behavior was determined using tensile testing at room temperature. Tensile tests were done according to ASTM E8M-04 standard with an Instron tensile testing machine at a cross-head speed of $2 \mathrm{~mm} \mathrm{~min}^{-1}$. The average strength of the three measurements was reported for each sample. In order to confirm the strength variation of martensite phase with cryogenic treatment, microhardness measurements were carried out using a Leitz RZD microhardness tester (Vickers indenter with a load of 150-g force).

\section{Result and discussion Microstructure}

The volume fractions of phases measured by metallography method are demonstrated in Fig. 4. As shown in this figure, the austenite volume fraction is $\sim 16.5 \%$ in the CHT sample, which is more than those in all DCT samples. With an increasing holding time of the deep cryogenic treatment, the austenite volume fraction remains unaltered while considering the error percentage. 


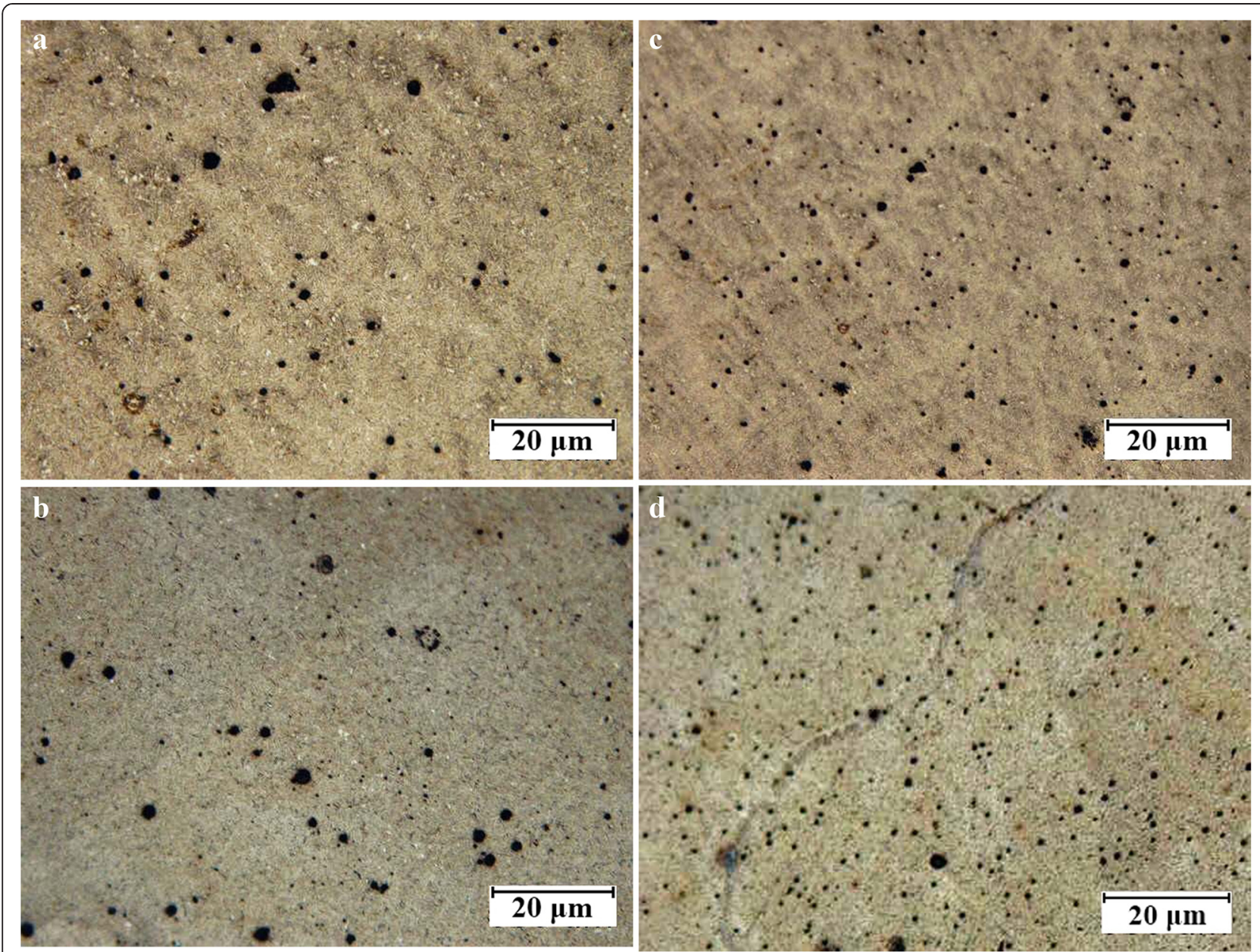

Fig. 3 Optical microstructures of a CHT, b DCT12, c DCT24, and d DCT48 steels showing distribution of carbides (dark regions)

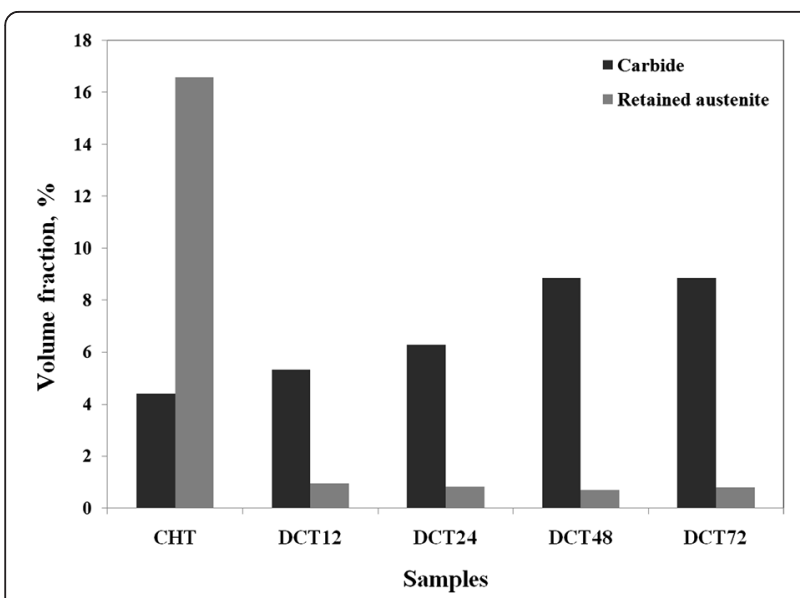

Fig. 4 Volume fractions (\%) of the constituent phases for $\mathrm{CHT}$ and DCT steels
This confirmed that the transformation of austenite to martensite is naturally not time dependent. According to Fig. 5, the (200) and (311) peaks of the retained austenite in the CHT sample can be observed, but this phase completely transformed to martensite and its percentage fell down the detection limit of the XRD techniques $(<1 \%)$. This evidence validated the data in Fig. 4 that shows variation of retained austenite volume fraction. Changes in carbide volume fractions of the studied microstructures are also shown in Fig. 4. It is clear from this figure that with the increasing holding time of the deep cryogenic treatment up to $48 \mathrm{~h}$ in the DCT steels, the carbide volume fraction increases and carbide particles are getting finer and more spherical (Fig. 3). This can be related to the precipitates thought to result from the migration of carbon atoms towards the dislocations during long holding at low temperatures. The carbon clouds around the dislocations serve as nuclei for the $\eta$-carbide, which is consistent with the previous studies by Oila et al. (2014) who observed precipitates of $\eta$-carbide in the vicinity of dislocations in the DCT martensite of a low-alloy medium-carbon steel. 


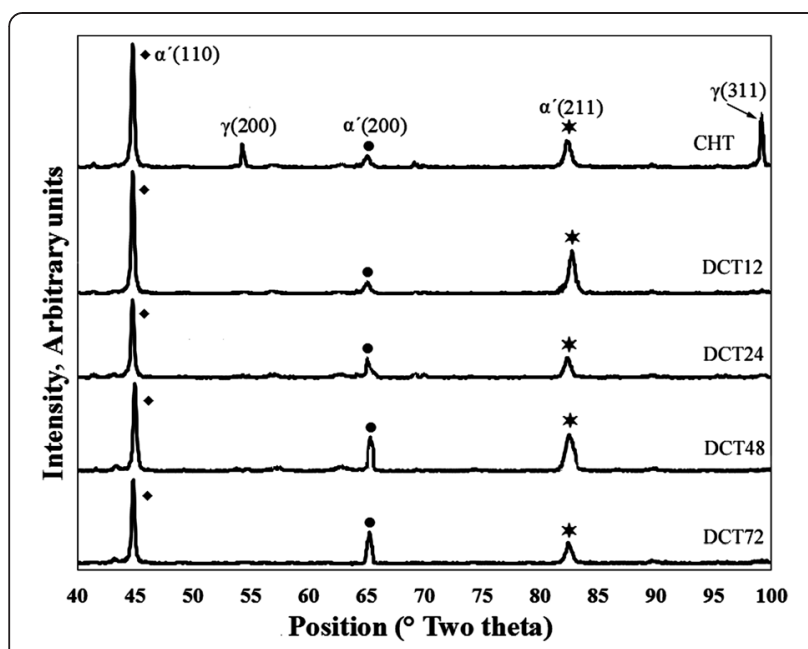

Fig. 5 XRD results of $C H T$ and DCT steels

The carbide nuclei can grow during the subsequent tempering. The thermodynamical driving force for the timedependent migration of carbon atoms from supersaturated martensite towards the dislocations is originated from the transformation stresses and thermal contraction during the deep cryogenic process. The thermal contraction is due to the cooling down to liquid nitrogen temperature. The stresses of martensitic transformation originated from the difference between volumes of crystal lattices of the origin austenite and produced martensite. This evidence injects internal stresses to the DCT steels. This thermodynamical driving force will decrease gradually (relaxation of internal stresses), with increasing the holding time up to $48 \mathrm{~h}$, which shows a plateau in $48 \mathrm{~h}$. By holding the DCT steels for more than the $48 \mathrm{~h}$ at the liquid nitrogen, no more $\eta$-carbide is nucleated, because there is not enough driving force to move the carbon atoms by the mechanism of stress-assisted diffusion (Tyshchenko et al. 2010).

It is pretended that carbides in the CHT steel are cementite while those in the DCT steels are $\eta$-carbide. This pretention can be proved as follows. (i) As can be seen from Fig. 3, carbides morphology of DCT48 microstructure is consisting of uniform distribution of small spherical particles, on the contrary of carbides that belong to the CHT microstructure. In other words, the carbides of the CHT microstructure are large blocky islands with non-uniform distribution. This observation proved that carbide types are different in the DCT steels from the CHT steel. (ii) The microhardness values of carbides for the investigated steels are shown in Fig. 6, which indicates that the CHT steel has carbides with microhardness values of $\sim 900 \mathrm{HV}$. While for the DCT steels, the microhardness values of carbides increased to $\sim 2020 \mathrm{HV}$ and remained unaltered for the different holding times of the deep cryogenic treatment. Higher order of microhardness values for the DCT steels

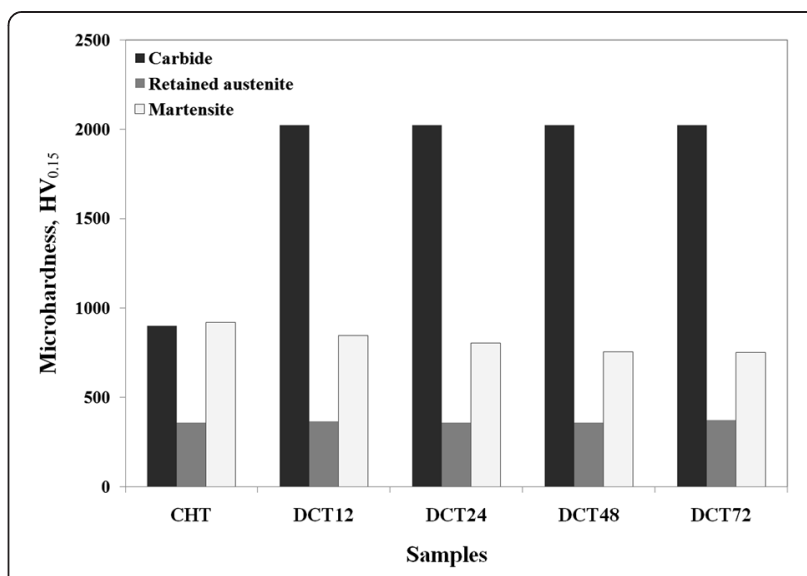

Fig. 6 Microhardness of the constituent phases for CHT and DCT steels

compared to that of the CHT steel can be seen to imply change in the type of carbides. According to microhardness measurements on carbide phase and what is mentioned by Oila et al. (2014), the carbides of DCT microstructure could be $\eta$-type with a higher order of microhardness magnitude while the carbides of CHT microstructure could be cementite. (iii) The microhardness results in Fig. 6 also indicate that the CHT steel has a greater microhardness value of martensite in comparison to those of the DCT steels. According to many studies (Chi et al. 2010; Jaswin and Lal 2011; Oila et al. 2014), microhardness of martensite is directionally proportional to its carbon content; thus, lower microhardness of martensite in DCT microstructures could be attributed to its lower carbon content. The decrement in carbon content of DCT martensite corroborates the idea of formation of $\eta$ carbides which absorbed more carbon atoms of martensitic matrix during the deep cryogenic process. (iv) TEM diffraction pattern of carbides of DCT48 steel is also given in Fig. 7c. It confirms that carbides are $\eta$-type $\left(\mathrm{Me}_{2} \mathrm{C}\right)$ in the DCT48 microstructure.

The typical TEM microstructures of the CHT and DCT48 steels are shown in Fig. 7. Before preparing TEM foils, the samples were not plastically deformed and just cryo-treated; thus, martensite as well as austenite phase have low density of dislocations. The dislocation density in the martensite phase adjacent to the $\eta$-carbide particles is low (Fig. 7b). Fig. 7a shows cementite particles which are located in the martensitic matrix. It is observed from this micrograph that the martensitic areas adjacent to the cementite involve high density of dislocations, unlike the martensite which is located close to the particles of $\eta$-carbides. Furthermore, the size of cementite $(\sim 320 \mathrm{~nm})$ in CHT steel becomes much larger in comparison to $\eta$-carbides $(210 \mathrm{~nm})$ in DCT48 microstructure. 


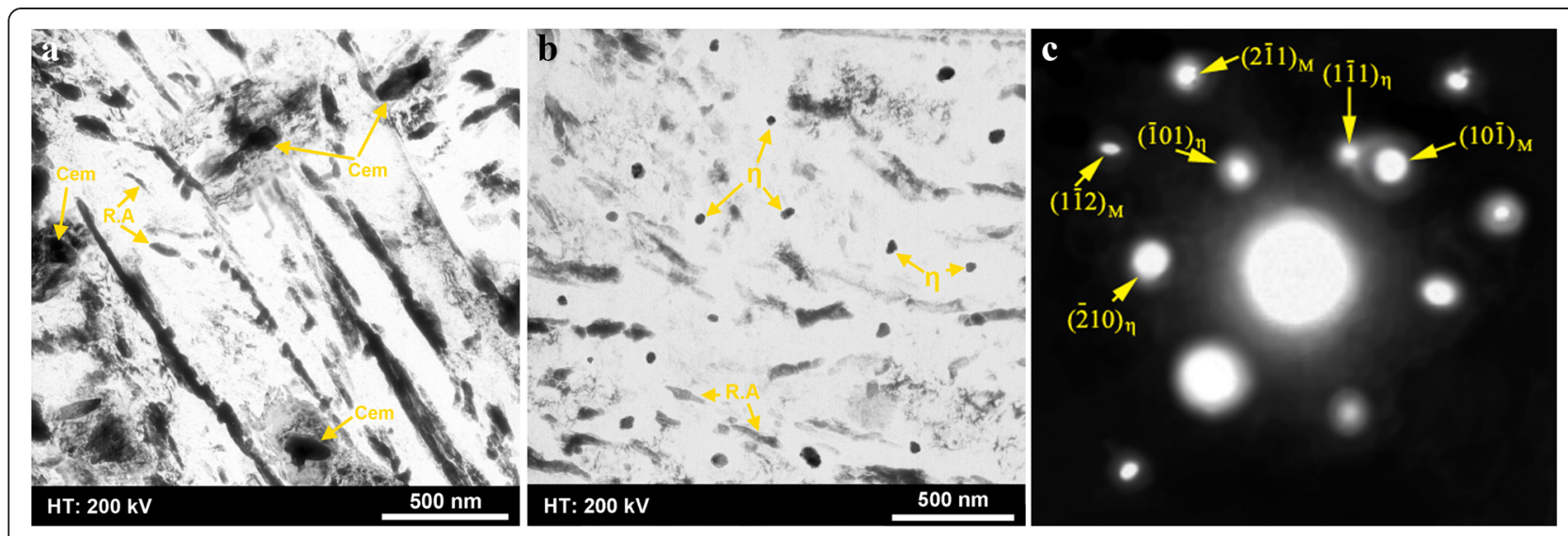

Fig. 7 TEM micrographs of $\mathbf{a}$ CHT and $\mathbf{b}$ DCT48 steels. $\mathbf{c}$ Electron diffraction of n-carbide in martensitic matrix of DCT48 steel; the incident beam direction is $(131)_{M}$ and $(121)_{\eta}$

\section{Strain hardening behavior}

Figure 8 shows the true stress-true strain curves on a double natural logarithmic scale, which were plotted for $\mathrm{CHT}$ and DCT steels with different holding times. As observed in Fig. 8, variation of $\ln \sigma$ with $\ln \varepsilon$ for the CHT steel is non-linear and obeys the two-stage strain hardening mechanism. The presence of different deformation micromechanisms makes distinct stages of strain hardening in the CHT steel. Lian et al. (1991), who said that hard phases deform elastically at the first stage and then deform partly elastically and partly plastically, proposed a model for multi-phase steels with hard and tough phases. It seems that the model of Lian et al. is applicable to the CHT steel since both hard and soft phases exist together. According to the model of Lian et al. on CHT steel with microstructure consisting

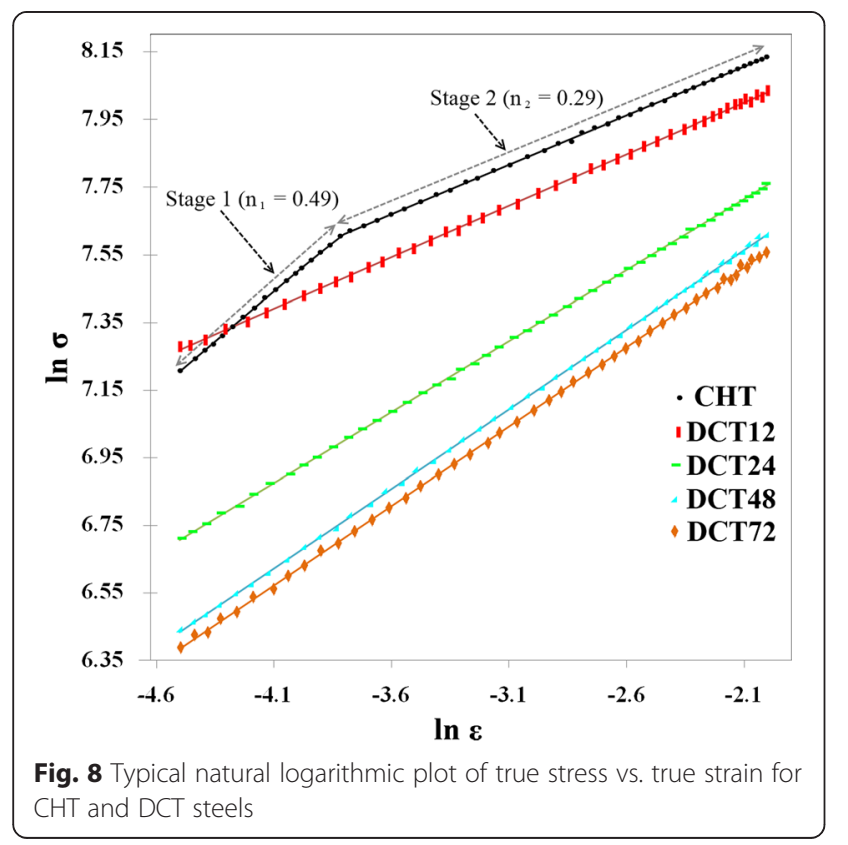

of martensite and austenite, the first stage can be related to the plastic deformation of austenite, and the second stage can be related to the co-deformation of both austenite and martensite. Martensite is naturally stronger than austenite (Fig. 6), so its plastic deformation begins during the second stage of strain hardening. At the second stage of strain hardening of CHT steel, martensite flow depends on its location. If it is located adjacent to austenite phase, when austenite strength reaches the yield strength of martensite due to strain hardening, martensite yields as a result of load transfer phenomenon in austenite-martensite interface (Zare and Ekrami 2011).

According to Fig. 8, the DCT steels show typical linear behavior of strain hardening. Linear variation of $\ln \sigma$ with $\ln \varepsilon$ indicates that the investigated DCT steels, with low volume fractions of retained austenite $(<1 \%)$, obey the one-stage strain hardening mechanism. At this stage, martensite deforms plastically and $\eta$-carbide particles could not deform because of their complex crystalline structure (orthorhombic). It should be mentioned that volume fractions of austenite are low enough $(<1 \%)$ (Figs. 4 and 5); this evidence has no significant effect on the plastic deformation of the DCT steels (Sakaki et al. 1990).

\section{The variation of strain hardening exponent}

As can also be seen from Fig. 8 for the CHT sample, the first stage has a high strain hardening exponent $\left(n_{1}\right)$ $(\sim 0.54)$ and the second stage $\left(n_{2}\right)$ has a low one $(\sim 0.21)$. The $n_{1}$ is a criterion for strain hardening of austenite, while $n_{2}$ is related to the co-strain hardening of austenite and martensite. According to more ductile nature of austenite in comparison with that of martensite, it is expected that the first stage has a greater strain hardening exponent compared to that of the second stage.

A comparison of strain hardening exponent of the CHT and DCT steels is shown in Fig. 9. Whereas 


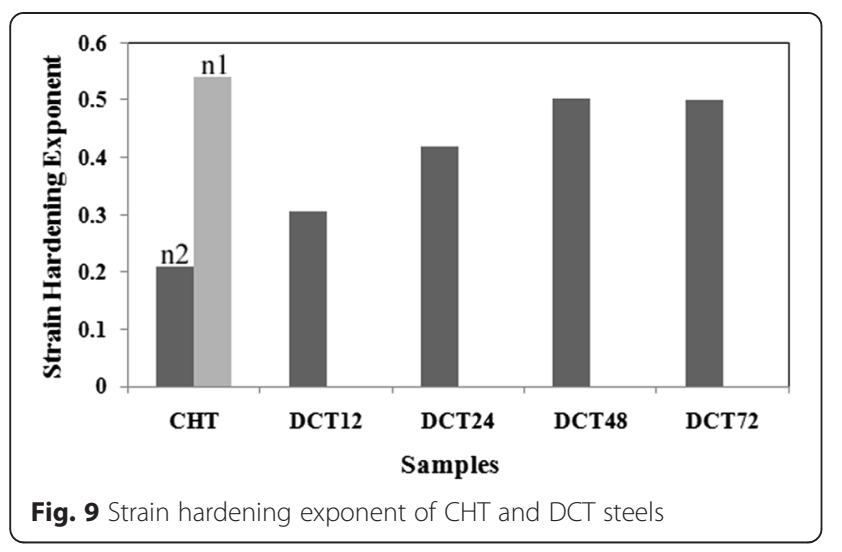

martensite deforms plastically at the second stage of strain hardening of the CHT sample, it is able to compare $n_{2}$ with the strain hardening exponent of the DCT steels. This is because the deformation micromechanism is strain hardening of martensite in both the CHT (at the second stage) and DCT steels. According to Fig. 9, $n_{2}$ for CHT steel is lower than strain hardening exponents of all DCT steels. This is due to the higher carbon content of the CHT steel compared to those of the DCT samples. The higher the carbon content of martensite, the lower its strain hardening ability ( $n$ ) (Krauss 2005). In the DCT steels, carbon atoms of martensitic matrix are absorbed by the formation of $\eta$-carbides and the low-carbon DCT martensite has a higher strain hardening ability compared to the high-carbon $\mathrm{CHT}$ martensite.

Change in strain hardening exponent with holding time of the deep cryogenic treatment is also shown in Fig. 9. The peaks/plateaus trend $(\sim 0.47)$ is observed in the variation of this parameter as a function of holding time at the deep cryogenic temperature. The variation of strain hardening exponent can be interpreted as well as explained for changes of UTS/YS and UTS-YS in the "Result and discussion" section to the "The variations of UTS/YS and UTS-YS" section.

\section{The variation of strength coefficient}

The change in the strength coefficient (as defined in Eq. 1) for the studied steels is illustrated in Fig. 10. According to this figure, the strength coefficient of the second stage of the CHT steel $(\sim 5900 \mathrm{MPa})$ is higher than those of the DCT steels. This evidence shows that the strength level for martensite plastic deformation of the CHT steel is higher than the strength level of the DCT steels. Results of microhardness on martensitic matrixes of the CHT and DCT steels (Fig. 6) confirm the higher strength level of the martensite within the CHT steels. The reason that the $K_{2}$ of the CHT steel is compared to

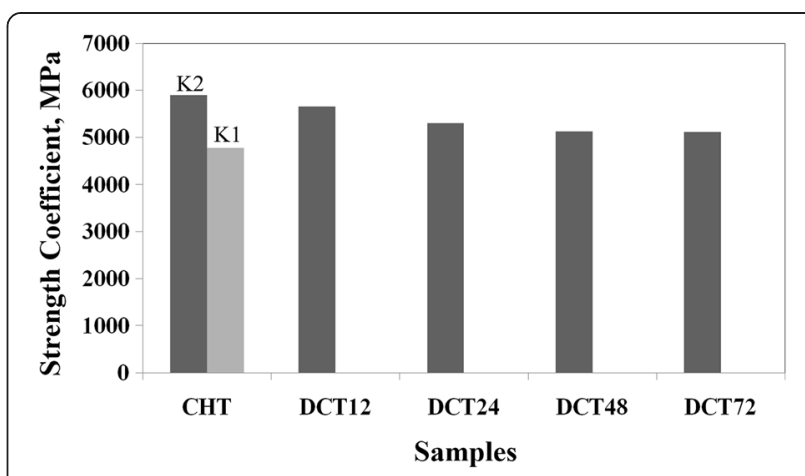

Fig. 10 Strength coefficient of CHT and DCT steels

the $K$ values of the DCT steels is that in the strain hardening stage 2 of the CHT steel, yielding of the martensite has activated micromechanism as well as the DCT steels.

It can also be seen in Fig. 10 that the strength coefficient of strain hardening stage 2 for the CHT steel is higher than that of stage 1 . This shows that at the second stage, a phase with a higher level of yield strength (martensite) is strain hardening in comparison to austenite phase which is deforming at the first stage of strain hardening. It should be mentioned that $K_{1}$ of the CHT steel is only affected by the flow behavior of austenite while $K_{2}$ is affected by two parameters which are as follows: (i) austenite deformation can be ongoing at strain hardening stage 2 , which leads to decrement in $K_{2}$ of CHT steel; and (ii) besides, as well as listed in Fig. 6, the existence of high-strength martensite in $\mathrm{CHT}$ steel and its deformation at the second stage of strain hardening, it compensates the decrement of the $K_{2}$ as a result of austenite plastic flow. Thus, this evidence is leading to a greater strength coefficient of stage 2 when compared to that of stage 1 .

As can be observed in Fig. 10, with the increasing holding time of the cryogenic process up to $48 \mathrm{~h}$, strength coefficient decreases and shows a plateau ( $5100 \mathrm{MPa})$. During deep cryogenic process, $\eta$-carbides precipitate with the mechanism of stress-assisted diffusion, so carbon content of martensite decreases and as a consequence its strength decreases (Totten and Howes 1997). So, with the increasing holding time up to $48 \mathrm{~h}$, more $\eta$-carbide nucleus forms and more carbon atoms are absorbed from martensitic matrix, and this evidence leads to decrement in strength coefficient. In holding times more than the optimum $(72 \mathrm{~h})$, the driving force for subzero diffusion (transformation and thermal stresses) decreases, and this leads to the invariable state of strength coefficient (Amini et al. 2012).

\section{The variations of UTS/YS and UTS-YS}

The variation of strain hardening exponent (Fig. 9) is also confirmed by the data in Fig. 11, which shows 


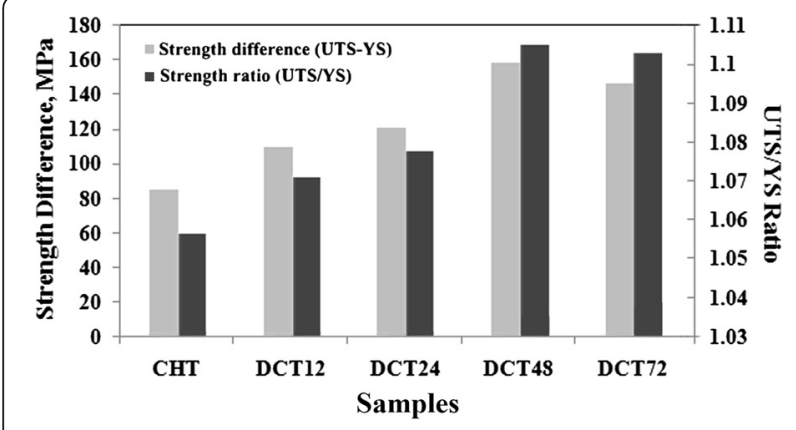

Fig. 11 Variations of UTS/YS and UTS-YS for CHT and DCT steels

peaks/plateaus in changes of UTS/YS and UTS-YS with the holding time of cryogenic treatment. The larger UTS/YS ratio and UTS-YS of the DCT steels compared to those of the CHT steel mean the greater capability of energy absorption before failure. In addition, larger deformations are experienced in which could serve as visible warning to structural occupants prior to final collapse. This evidence is highly important in airplane components.

Increasing trend of the UTS/YS and UTS-YS with the holding time of the deep cryogenic treatment up to $48 \mathrm{~h}$ is also shown in Fig. 11. The peaks/plateaus trend of these parameters has been identified as one of the manifestation of $\eta$-carbide formation. With increasing holding time up to the peak value ( $48 \mathrm{~h}$ ), more carbon atoms diffuse to form $\eta$-carbides. So with the formation of $\eta$ carbides in martensitic matrix, carbon content of martensite decreases and its stretch formability increases, which means enhancement in UTS/YS and UTS-YS as well as the strain hardening exponent $(n)$ (Totten and Howes 1997). Also, with an increasing holding time more than $48 \mathrm{~h}$, the UTS/YS and UTS-YS remained unaltered. It revealed that after $48 \mathrm{~h}$, the internal stresses decrease to diffuse carbon atoms and unaltered trend in the UTS/YS and UTS-YS parameters (Amini et al. 2012).

\section{Conclusions}

The main conclusions emerging from the relationship between the holding time of the deep cryogenic treatment and strain hardening parameters as well as microstructural changes investigated in this paper are the following:

1. Completion of martensitic transformation and $\eta$-carbides precipitation occur during cryogenic treatment. An increment in the degree of holding time of the deep cryogenic treatment up to $48 \mathrm{~h}$ significantly increases the volume fraction of the $\eta$-carbides and their uniformity while decreases the particle size of $\eta$-carbides. In longer holding durations more than $48 \mathrm{~h}$, the volume fraction, uniformity, and particle size of carbides do not change anymore in deep cryo-treated steels. Precipitation of homogenized $\eta$-carbides within DCT martensite causes decrement in carbon concentration of the martensite as compared to that in the CHT steel. The results also show that exhaustion of carbon atoms from martensitic matrix and the precipitation of $\eta$-carbide are increased with the increasing holding time of the deep cryogenic treatment up to $48 \mathrm{~h}$.

2. Using the diffraction patterns of TEM, the existence of $\eta$-carbides is confirmed in the deep cryo-treated steels. TEM micrograph of the CHT steel indicates high density of dislocations within the interphase boundaries of cementite/martensite, while the deep cryo-treated sample for $48 \mathrm{~h}$ contains $\eta$-carbides within low-carbon martensitic matrix. Additionally, $\eta$-carbide which forms during deep cryogenic treatment has homogenized spherical morphology as compared to the carbide of the CHT sample.

3. Strain hardening of CHT steel comprises two stages with different exponents. The higher strain hardening exponent $(\sim 0.54)$ at the first stage is attributed to the deformation of the austenite, and the lower strain hardening exponent $(\sim 0.21)$ at the second stage is associated with the plastic deformation of the both austenite and martensite.

4. The DCT steels show one-stage strain hardening behavior. At this stage of strain hardening, martensite phase deforms plastically.

5. By increasing the holding time of the deep cryogenic treatment, the strain hardening exponent shows peaks/plateaus $(\sim 0.47)$, while strength coefficient has a decreasing plateau trend. The variations of Hollomon equation parameters with holding time at the cryogenic temperature are related to the microstructural changes including precipitation of $\eta$-carbides and decrement in carbon content of martensite phase during cryogenic treatment.

6. The variation of Hollomon' strain hardening exponent is confirmed by the changes of UTS/YS ratio and UTS-YS as the criteria for strain hardening.

\section{Competing interest}

The authors declare that they have no competing interests.

\section{Authors' contributions}

AZ carried out primary studies, tensile tests and microstructural observations. $\mathrm{HM}$ assisted for preparation of all experiments. HM and SRH contributed to write and edit the manuscript. All authors read and approved the final manuscript.

Received: 2 August 2015 Accepted: 9 November 2015 Published online: 14 November 2015

\section{References}

Akbarpour, MR, \& Ekrami, A. (2008). Effect of ferrite volume fraction on work hardening behavior of high bainite dual phase (DP) steels. Materials Science and Engineering A, 477, 306-310. 
Akhbarizadeh, A, Golozar, M, Shafeie, A, \& Kholghy, M. (2009). Effects of austenizing time on wear behavior of D6 tool steel after deep cryogenic treatment. Journal of Iron and Steel Research International, 16, 29-32.

Amini, K, Akhbarizadeh, A, \& Javadpour, S. (2012). Effect of deep cryogenic treatment on the formation of nano-sized carbides and the wear behavior of D2 tool steel. Journal of Minerals, Metallurgy, and Materials, 19, 795-799.

Chi, HX, Ma, DS, Yong, QL, Wu, LZ, Zhang, ZP, \& Wang, YW. (2010). Effect of cryogenic treatment on properties of Cr8-type cold work die steel. Journal of Iron and Steel Research International, 17, 43-59.

Fridman, YB. (1975). Mechanical properties of metals. Met Science Heat Treat, 17, 637.

Girault, E, Jacques, P, Harlet, P, Mols, K, Van Humbeeck, J, \& Aernoudt, E. (1998). Metallographic methods for revealing the multiphase microstructure of TRIP-assisted steels. Materials Characterization, 40, 111-118.

Hadi, A. (2014). MS thesis, Maleke-ashtar University of Technology, Isfahan, Iran (in Persian). Hertzberg, RW. (1996). Deformation and fracture mechanics of engineering materials. New York: Wiley.

Hollomon, JH. (1945). Tensile deformation. AIME Trans, 12, 1-22.

Jaswin, MA, \& Lal, DM. (2011). Effect of cryogenic treatment on the tensile behaviour of En 52 and 21-4 N valve steels at room and elevated temperatures. Materials and Design, 32, 2429-2437.

Klopp, W. (1992). Aerospace structural metals handbook (2nd ed., pp. 1-12).

Koneshlou, M, Meshinchi Asl, K, \& Khomamizadeh, F. (2011). Effect of cryogenic treatment on microstructure, mechanical and wear behaviors of AISI H13 hot work tool steel. Cryogenics, 51, 55-61.

Krauss, G (2005). Steels processing, structure, and performance (pp. 127-136). Materials Park, Ohio, U.S.A: ASM International.

Leskovšek, V, Kalin, M, \& Vižintin, J. (2006). Influence of deep-cryogenic treatment on wear resistance of vacuum heat-treated HSS. Journal of Vacuum Science and Technology A, 80, 507-518

Li, S, Deng, L, Wu, X, Min, YA, \& Wang, H. (2010a). Influence of deep cryogenic treatment on microstructure and evaluation by internal friction of a tool steel. Cryogenics, 50, 754-758.

Li, S, Deng, L, Wu, X, Wang, H, Min, YA, \& Min, N. (2010b). Effect of deep cryogenic treatment on internal friction behaviors of cold work die steel and their experimental explanation by coupling model. Materials Science and Engineering A, 527, 7950-7954.

Li, S, Min, N, Deng, L, Wu, X, Min, YA, \& Wang, H. (2011). Influence of deep cryogenic treatment on internal friction behavior in the process of tempering. Materials Science and Engineering A, 527, 1247-1250.

Lian, J, Jiang, Z, \& Liu, J. (1991). Theoretical model for the tensile work hardening behaviour of dual-phase steel. Materials Science and Engineering A, 147, 55-65.

Marin, J (1962). Mechanical behavior of engineering materials. Englewood Cliffs (pp. 19-23). N.J.: Prentice-Hall.

Meyers, M, \& Chawla, K. (1991). Mechanical behavior of materials. Materials Science and Engineering, 132, 1-11.

Oila, A, Lung, C, \& Bull, S. (2014). Elastic properties of eta carbide ( $\eta$-Fe2C) from ab initio calculations: application to cryogenically treated gear steel. Journal of Materials Science, 49, 2383-2390.

Sakaki, T, Ohnuma, K, Sugimoto, K, \& Ohtakara, Y. (1990). Plastic anisotropy of dual-phase steels. International Journal of Plasticity, 6, 591-613.

Senthilkumar, D, Rajendran, I, Pellizzari, M, \& Siiriainen, J. (2011). Influence of shallow and deep cryogenic treatment on the residual state of stress of 4140 steel. Journal of Materials Processing Technology, 211, 396-401.

Swift, HW. (1952). Plastic instability under plane stress. Journal of the Mechanics and Physics Solids, 1, 1-18.

Totten, GE, \& Howes, MA (1997). Steel heat treatment handbook (2nd ed., pp. 231-253). Portland, Oregon, U.S.A.: CRC Press.

Tyshchenko, A, Theisen, W, Oppenkowski, A, Siebert, S, Razumov, O, \& Skoblik, A. (2010). Low-temperature martensitic transformation and deep cryogenic treatment of a tool steel. Materials Science and Engineering A, 527, 7027-7039.

Unterweiser, PM (1982). Heat treater's guide: standard practices and procedures for steel (pp. 39-46). First edition, Second printing edition. Materials Park, Ohio, U.S.A.: ASM International.

Voce, E. (1948). The relationship between stress and strain for homogeneous deformation. Journal of the Institute of Metals, 74, 537-562.

Zare, A, \& Ekrami, A. (2011). Effect of martensite volume fraction on work hardening behavior of triple phase (TP) steels. Materials Science and Engineering A, 528, 4422-4426.

\section{Submit your manuscript to a SpringerOpen ${ }^{\odot}$ journal and benefit from:}

- Convenient online submission

- Rigorous peer review

- Immediate publication on acceptance

- Open access: articles freely available online

- High visibility within the field

- Retaining the copyright to your article

Submit your next manuscript at $\gg$ springeropen.com 